\title{
Study of the multiplicity distributions in relativistic nucleus - nucleus collisions using the multiplicity distribution moments method
}

Catalin Ristea $^{1 \mathrm{a}}$, Alexandru Jipa ${ }^{1}$, Oana Ristea ${ }^{1}$, Calin Besliu ${ }^{1}$, Ionel Lazanu ${ }^{1}$, Marius Calin ${ }^{1}$, Tiberiu Esanu $^{1}$ and Vanea Covlea ${ }^{1}$

${ }^{1}$ Atomic and Nuclear Physics Chair, Department of Structure of Matter, Atmosphere and Earth Physics, Astrophysics, Faculty of Physics, University of Bucharest, Romania

\begin{abstract}
In this work, we will present beam energy and system size dependence of the higher order moments (skewness and kurtosis) of net-kaon and total proton multiplicity distributions for $\mathrm{Au}+\mathrm{Au}$ collisions simulated using the UrQMD code.
\end{abstract}

\section{Introduction}

One of the major tasks in our understanding of the QCD phase diagram is locating the QCD critical point $\mathrm{CP}$ (connecting the first-order boundary separating the hadronic from the partonic matter at high density with the cross-over boundary at low density). Experimentally, we can probe this phase diagram with heavy ion collisions at different energies where the matter is heated and compressed. The system created in such collisions follows different trajectories on the temperature $(\mathrm{T})$ and baryon chemical potential $\left(\mu_{\mathrm{B}}\right)$ plane during its time evolution.

The characteristic signature of the existence of a $\mathrm{CP}$ is an increase of fluctuations [1]. The fluctuations of the charge/baryon number/strangeness can be related to event-by-event moments of various observables in heavy-ion collisions. The experimentally measurable net-proton number (number of anti-protons substracted from the number of protons) fluctuations may reflect the fluctuations of the net-baryon number at CP. It is expected that the presence of fluctuations from the critical point may lead to a non-Gaussian shape in the event-by-event multiplicity distributions and non-monotonic behavior of the observables studied (such as higher order moments) [2].

Therefore, higher moments (skewness and kurtosis) of multiplicity distributions are proposed to provide one of the most sensitive probes towards the search for the $\mathrm{CP}$ because are conjectured to reflect the large fluctuations associated with the hadron-quark phase transition. For example, a sign change in the skewness or kurtosis may be an indication that the system crossed the phase boundary [3]. In the last years the multiplicity moments have been intensively studied both experimentally [4-6] and theoretically [7-9].

The skewness and kurtosis of the event-by-event multiplicity distributions are defined as:

$$
S=\frac{\left\langle(N-\langle N\rangle)^{3}\right\rangle}{\sigma^{3}}
$$

\footnotetext{
${ }^{\mathrm{a}}$ Corresponding author: catalin.ristea@live.com
} 


$$
\kappa=\frac{\left\langle(N-\langle N\rangle)^{4}\right\rangle}{\sigma^{4}}-3
$$

where $\mathrm{N}$ is the particle number (multiplicity) in one event and $\langle\mathrm{N}\rangle$ is the averaged particle multiplicity over the whole event ensemble. We analyzed the net-kaon multiplicities $\left(N_{K^{+}-K^{-}}\right.$ $=N_{K^{+}}-N_{K^{-}}$, which is the positive kaon number minus the negative kaon number) and total proton multiplicities $\left(N_{p+\bar{p}}=N_{p}+N_{\bar{p}}\right.$, which is the sum of the proton and antiproton number) from $\mathrm{Au}+\mathrm{Au}$ collisions at various energies. The aim of the present study is to have a baseline for the case of the absence of the critical point, since the UrQMD code does not have the physics of the critical point.

\section{Results}

This work presents a study of the moments of net-kaon and total protons multiplicity distributions in $\mathrm{Au}+\mathrm{Au}$ collisions at different energies (at energies that will be available at CBM-FAIR: $\mathrm{p}_{\text {lab }}=2$ $\mathrm{GeV} / \mathrm{c}, 11 \mathrm{GeV} / \mathrm{c}, 25 \mathrm{GeV} / \mathrm{c}$ and at RHIC energies: $\sqrt{S_{N N}}=62.4$ and $200 \mathrm{GeV}$ ) using UrQMD (Ultrarelativistic Quantum Molecular Dynamics) code [10].

The centrality dependence of the event-by-event net-kaon $\left(\mathrm{N}_{\mathrm{K}}{ }^{+}-\mathrm{N}_{\mathrm{K}}{ }^{-}\right)$distributions in $\mathrm{Au}+\mathrm{Au}$ collisions at $\mathrm{p}_{\mathrm{lab}}=25 \mathrm{GeV} / \mathrm{c}$ is shown in the Fig.1. Going from peripheral to central collisions, it is found that the event-by-event net-kaon distributions become wider and more symmetric for central collisions. Also, for peripheral collisions the mean is shifted towards zero. These event-by-event distributions for each centrality were used to compute higher order moments (3rd moment - skewness and 4th moment - kurtosis). The moments are decreasing from peripheral to central events.
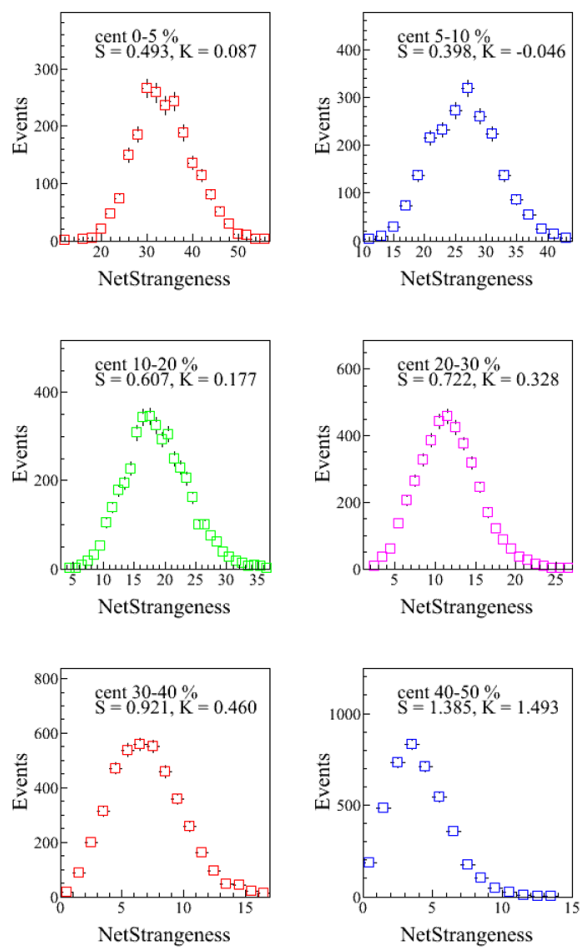

Figure 1. The centrality dependence of the net-Kaon multiplicity distributions from UrQMD code in $\mathrm{Au}+\mathrm{Au}$ collisions at $\mathrm{p}_{\mathrm{lab}}=25 \mathrm{GeV} / \mathrm{c}$. 
The two higher order moments ( $\mathrm{S}$ and $\mathrm{K}$ ) which describe the shape of the net-kaons distributions in $\mathrm{Au}+\mathrm{Au}$ collisions at various collision energies are plotted as a function of the number of participants, $\mathrm{N}_{\text {part }}$.
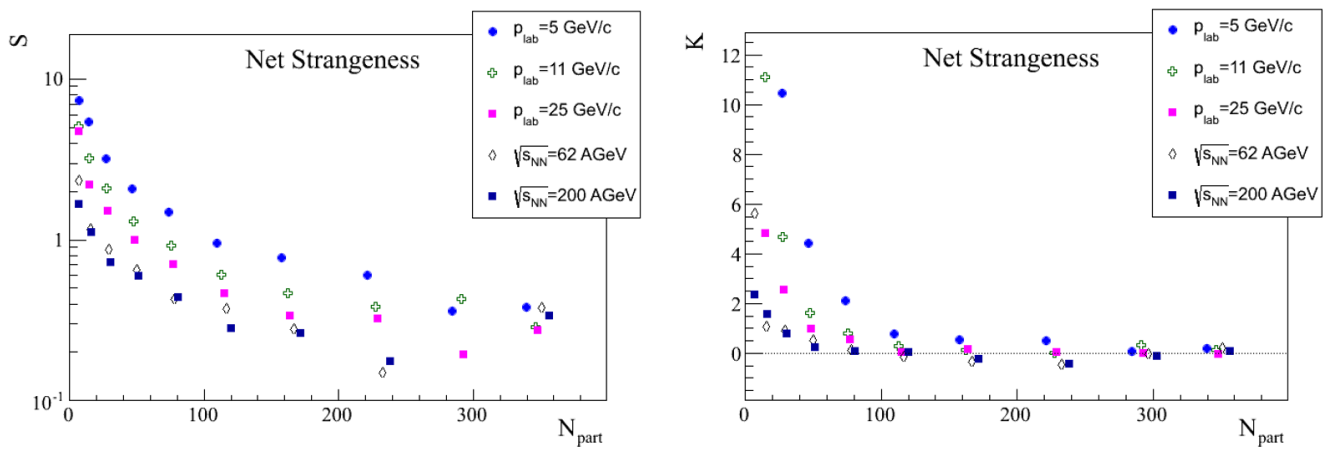

Figure 2. The centrality dependence of the net-Kaon skewness (left panel) and kurtosis (right panel) in $\mathrm{Au}+\mathrm{Au}$ collisions at different energies simulated using UrQMD code.

For all studied energies, the skewness is positive and decreases as the number of participants increases. For the same collision centrality ( same $\mathrm{N}_{\text {part }}$ ), skewness values decrease as the beam energy increases. We also note that for the same collision centrality ( same $\mathrm{N}_{\text {part }}$ ), the asymmetry parameter varies stronger with the energy especially for peripheral collisions.

The $4^{\text {th }}$ moment of the net kaon multiplicity distributions (kurtosis) is close to 0 value in central collisions and we observe that deviations from zero get larger in more peripheral collisions (right panel of the Fig. 2). The skewness $\mathrm{S}$ has stronger energy dependence than that of kurtosis $\kappa$, for central $\mathrm{Au}+\mathrm{Au}$ collisions.
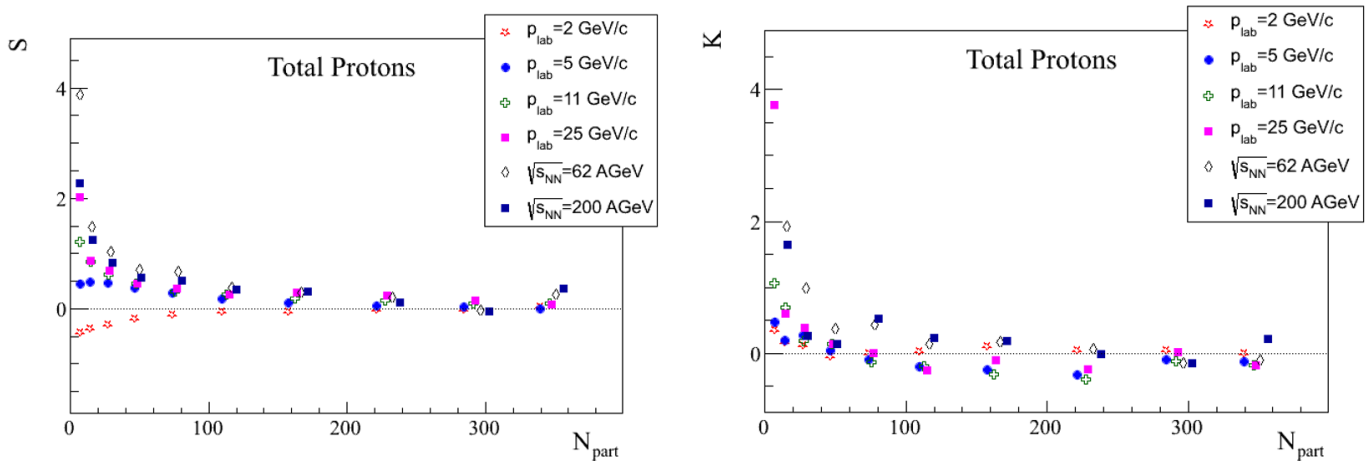

Figure 3. The centrality dependence of the total proton skewness (left panel) and kurtosis (right panel) in $\mathrm{Au}+\mathrm{Au}$ collisions at different energies simulated using UrQMD code.

For the total proton event-by-event distributions, the skewness has weaker energy dependence in more central collisions, but clearer energy dependence for peripheral collisions. Above $5 \mathrm{GeV} / \mathrm{c}, \mathrm{S}$ is positive in peripheral collisions, and increase towards more peripheral collisions. Below $5 \mathrm{GeV} / \mathrm{c}, \mathrm{S}$ turns to be negative in peripheral collisions. We observe that for the lowest energy considered, the behaviour is different, which might indicate a change in the behaviour of the system formed in the collision.

The total proton kurtosis has weak centrality and energy dependence. The kurtosis of total-proton in peripheral collisions is found to be always positive at all measured energies. In central collisions, its value is around zero at all incident energies. 


\section{Conclusions}

In this work, the higher order moments of net-kaon and total proton multiplicity distributions from $\mathrm{Au}+\mathrm{Au}$ collisions simulated using the UrQMD code in a broad energy range and different system sizes have been presented. The energy dependence of the moments is stronger in peripheral collisions, both for net-kaons and total protons. For the same collision centrality, the skewnes and kurtosis values for net-kaons decrease with increasing energy. In the case of total protons, for the same collision centrality, the skewnes and kurtosis values increase with beam energy. Present results are relevant for the absence of critical point (as extracted from UrQMD code) and it would be interesting to be compared with future experimental data at SIS-100.

\section{Acknowledgments}

The work of Oana Ristea and Catalin Ristea was supported by the strategic grant POSDRU/89/1.5/S/58852, Project Postdoctoral programme for training scientific researchers, cofinanced by the European Social Found within the Sectorial Operational Program Human Resources Development 2007-2013. This work was partially supported by PN-II-ID-PCE-IDEI 34/05.10.2011 grant.

\section{References}

1. C. Athanasiou, K. Rajagopal and M. Stephanov, Phys. Rev. D 82, 074008 (2010); M. A. Stephanov, Int. J. Mod. Phys. A 20, 4387 (2005); Z. Fodor et al., JHEP 0404, 50 (2004).

2. Y. Hatta and M. A. Stephanov, Phys. Rev. Lett. 91, 102003 (2003); X. Luo et al., arXiv:1105.5049.

3. M. Asakawa et al., Phys. Rev. Lett. 103, 262301 (2009).

4. M. Aggarwal, et al., STAR Collaboration, Phys. Rev. Lett. 105, 022302 (2010).

5. T. J. Tarnowsky for STAR Collaboration, J.Phys. G 38, 124054 (2011).

6. B. Mohanty, J. Phys. G 38, 124023 (2011).

7. F. Karsch and K. Redlich, Phys. Lett. B 695, 136 (2011).

8. B. Friman, F. Karsch, K. Redlich, V. Skokov, Eur.Phys.J. C 71, 1694 (2011).

9. D.J. Wang, L.P. Csernai, D. Strottman, Cs. Anderlik, Y. Cheng, D.M. Zhou, Y.L. Yan, X. Cai, B.H. Sa, Eur.Phys.J. A 48, 168 (2012).

10. S.A.Bass et al., Prog.Part.Nucl.Phys. 41, 225 (1998); M.Bleicher et al., J.Phys. G 25, 1859 (1999) 